\title{
Systemic arterial aneurysms in a 5 month old male with severe refractory kawasaki's disease
}

\author{
Benson Hsu ${ }^{1}$, Ben Soriano ${ }^{2}$, M. Shahirar Salamet ${ }^{2}$, Scott B. Reeder ${ }^{3}$ and J. Carter Ralphe ${ }^{1 *}$ \\ ${ }^{1}$ Department of Pediatrics, University of Wisconsin School of Medicine \& Public Health Madison, WI, USA \\ ${ }^{2}$ Department of Pathology, University of Wisconsin School of Medicine \& Public Health Madison, WI, USA \\ ${ }^{3}$ Department of Radiology, University of Wisconsin School of Medicine \& Public Health Madison, WI, USA
}

\begin{abstract}
This is the presentation of a 5 month old infant male initially diagnosed with atypical Kawasaki's disease who developed disseminated coronary artery dilation and multiple aneurysms in mid-size systemic arteries. He suffered an acute bleeding event and later died. This case underscores the possibility of systemic arterial involvement in severe cases of Kawasaki's disease and raises the issue whether wider screening for extracardiac vascular abnormalities might be indicated.
\end{abstract}

Abbreviations: CRP: c-reactive protein, ESR: erythrocyte sedimentation rate, EKG: electrocardiogram, OR: operating room, PICU: pediatric intensive care unit

\section{Introduction}

Kawasaki's disease is a self-limited vasculitis predominately occurring in children under the age of five, more often in males of Asian descent. Diagnosis is by meeting four of five clinical criteria in a patient with fever greater than five days duration. Criteria include changes of the extremities (peripheral edema, peripheral erythema, or periungal desquamation,) bilateral conjunctival injection, changes in the mucus membrane of the upper respiratory tract, polymorphous rash, and cervical adenopathy (usually unilateral). Although laboratory studies can be useful in supporting the diagnosis of Kawasaki's disease, they are not required. Common findings include leukocytosis, anemia, thrombocytosis, elevated acute phase reactants (ESR and CRP,) elevated transaminases, hyperbilirubinemia, hypoalbuminia, and sterile pyuria. Analysis of cerebral spinal fluid may demonstrate pleocytosis. Imaging studies are required to evaluate for coronary artery involvement including aneurysms or diffuse dilation, or simply peri-vascular enhancement which can occur in $15-25 \%$ of untreated cases. When coronary artery abnormalities are identified but additional clinical criteria are not met, a diagnosis of incomplete Kawasaki's can be made [1]. Systemic midsize artery involvement has been rarely reported [2].

\section{Patient presentation}

The patient is a five month old male twin, born premature at 32 weeks gestation, who presented with a 5 day history of fever, rash, and irritability. He had an elevated CRP, leukocytosis, anemia, and thrombocytopenia. He did not fulfill classic criteria and was diagnosed with incomplete Kawasaki's Disease. Initial echocardiogram on admission demonstrated nonspecific coronary ectasia and perivascular brightness. Following two courses of intravenous immunoglobulin, patient defervesced and was discharged home on high dose aspirin. That evening, he redeveloped fever and was readmitted. Follow-up echocardiogram now revealed diffuse severe coronary dilation with giant coronary aneurysms $(>8 \mathrm{~mm})$ involving the left main, left anterior descending, left circumflex, right main, and right posterior descending coronary arteries with a small pericardial effusion. He received a second dose of intravenous immunoglobulin. That evening he developed ST segment changes on EKG concerning for myocardial ischemia prompting transfer to the PICU and commencement of heparin while Coumadin was started. Over the next several days fever persisted for which he received a fourth dose of intravenous immunoglobulin, steroids and infliximab. Pulsatile masses were noted in the axillae and groin. Whole body time resolved MR angiography [3] showed multiple lobulated masses bilaterally in the axillary, brachial, and iliac regions (Figure 1A) that was confirmed by ultrasound to be arterial aneurysms.
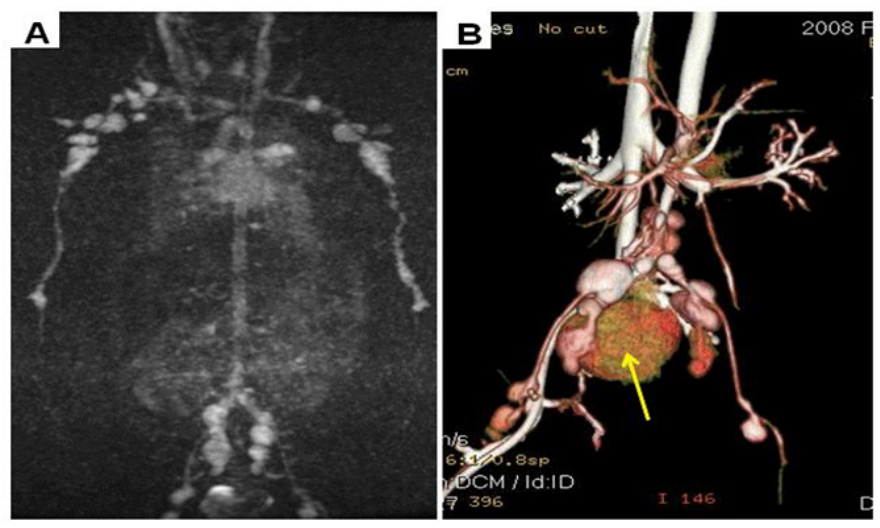

Figure 1. A. Maximum intensity projection (MIP) images from a time resolved MR angiogram, acquired after $2 \mathrm{ml}$ injection of gadobenate dimeglumine, demonstrates innumerable enhancing lobulated masses in the axillary, brachial, iliac and femoral arteries, consistent with severe aneurysmal disease related to Kawasaki's. B. Volume rendered contrast enhanced CT angiogram of the abdomen and pelvis demonstrates iliac and femoral artery aneurysms and also show areas of active extravasation of iodinated contrast (arrow) into the pelvis, caused by an acutely ruptured aneurysm.

Correspondence to: J. Carter Ralphe, MD Assistant Professor of Pediatrics University of Wisconsin School of Medicine \& Public Health H4/414 Clinical Sciences Center 600 Highland Avenue Madison, WI, USA, Tel: 608-262-1603; Fax: 265-8065; E-mail: jcralphe@pediatrics.wisc.edu

Key words: Vasculitis, arterial aneurysms, Kawasaki disease

Received: June 22, 2016; Accepted: July 08, 2016; Published: July 12, 2016 
No involvement of the thoracic or abdominal aorta or renal arteries was noted. On the eleventh day of his PICU hospitalization, the patient acutely decompensated and required cardiopulmonary resuscitation. An echocardiogram showed no evidence of tamponade. An emergent $\mathrm{CT}$ scan revealed active bleeding from the right common iliac artery (Figure 1B) and the patient was taken immediately to the OR for repair. Intraoperative findings confirmed a ruptured right common iliac aneurysm that was ligated and oversewn. After returning to the PICU postoperatively, the patient continued to require repeated cardiopulmonary resuscitation. Care was withdrawn at parents' request and the patient died soon after.

Post-mortem exam confirmed the extensive dilation and giant aneurysms in both the right and left coronary circulation (Figure 2 A-C). Multiple saccular aneurysms involving the brachial, common iliac, and femoral arteries were confirmed with a large amount of clotted blood in the pelvis. Histological sections from involved arteries revealed $\mathrm{CD} 3+/ \mathrm{CD} 20$ - lymphocytic infiltrate consistent with a T-cell mediated vasculitis that is consistent with the late vasculitis of Kawasaki's Disease (Figure 2D) [4].

\section{Discussion}

The pathogenesis of Kawasaki's disease remains unclear. A prior infection may be a predisposing factor although cultures in patients with Kawasaki's disease are generally negative. Historically, younger, male infants with atypical or incomplete disease have a higher risk of
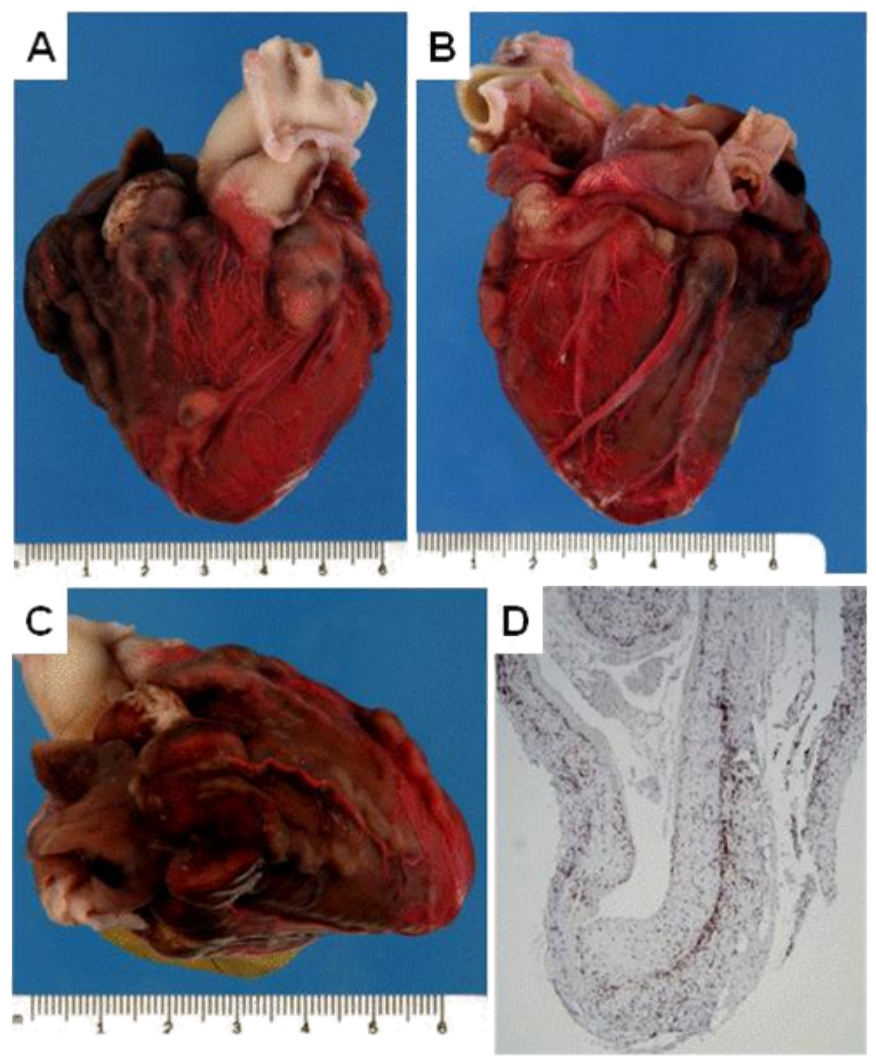

Figure 2. A: Anterior aspect of heart revealing diffuse dilation of coronary vessels with ectasia of the circumflex artery and giant aneurysm of the proximal left anterior descending artery. B: Posterior aspect of heart demonstrating severe dilation of right coronary and posterior descending arteries with diffuse involvement of major branches. C: Lateral aspect of heart showing severe enlargement of anterior descending and branches with significant tortuosity. D: Histological cross section of representative aneurysmal coronary vessel stained with anti-CD3 antibody (brownish staining cells). morbidity and mortality. Standard therapy includes one to two doses of intravenous immunoglobulin, followed by initiation of long-term aspirin therapy to prevent or treat the major long-term complication of coronary artery dilation and subsequent stenosis [5]. Treatment of refractory Kawasaki's disease (those having failed two doses of intravenous immunoglobulin) is controversial. Proposed agents include steroids, monoclonal antibody against pro-inflammatory cytokines (such as infliximab, a monoclonal antibody against tumor necrosis factor alpha,) and cytotoxic agents (either cyclophosphamide or methotrexate)

This case demonstrates a particularly severe form of Kawasaki's disease vasculitis. Review of the literature finds no documented extracoronary mid-sized arterial involvement in Kawsaki's disease. The typical vasculitis contributing to the palmar erythema, desquamation, conjunctivitis, and tongue changes has been reported to lead to tissue necrosis [6]. In our case, this suspicion arose only during a careful and thorough exam, something often compromised in severely ill, irritable infants, that identified pulsatile masses in the axillae and groin. Without data, and in the face of evolving ECG changes, we managed this child acutely with heparin anticoagulation while starting Coumadin, and aspirin antiplatelet therapy. He also received one dose of clopidogrel. At the time our concerns for coronary thrombosis outweighed those for possible bleeding complications, though the high overall risk was understood.

We do not know what other factors might have contributed to this unusual presentation. Viral-like inclusion bodies were found in the lungs of this patient similar to findings in other patients with KD and supporting an underlying viral etiology [7]. Increasing evidence suggests that an individual's immune response to the inciting event or pathogen, as determined by their genetic background, exerts a significant influence on the severity of presentation and responsiveness of the illness to IVIG $[8,9]$. As the era of personalized medicine gains support, inflammatory diseases such as KD may benefit from the development of tools to precisely define the risk for severe/refractory disease, and allow practitioners to tailor treatment regimens with better precision.

The potential for extra-coronary arterial involvement in Kawasaki's Disease underscores the importance of careful examination and index of suspicion for systemic vascular involvement in severe presentations. While aneurysms of the systemic arteries are likely quite rare, their incidence and natural history cannot be known unless they are actively identified.

\section{Disclosures}

The authors have no conflicts to disclose.

\section{References}

1. Newburger JW, Takahashi M, Gerber MA, Gewitz MH, Tani LY, et al. (2004) Diagnosis, Treatment, and Long-Term Management of Kawasaki Disease: A Statement for Health Professionals From the Committee on Rheumatic Fever, Endocarditis and Kawasaki Disease, Council on Cardiovascular Disease in the Young, American Heart Association. Circulation 110: 2747-2771. [Crossref]

2. Dajani AS, Taubert KA, Gerber MA, Shulman ST, Ferrieri P, et al. (1993) Diagnosis and therapy of Kawasaki disease in children. Circulation 87: 1776-80. [Crossref]

3. Korosec FR, Frayne R, Grist TM, Mistretta CA (1996) Time-resolved contrastenhanced 3D MR angiography. Magn Reson Med 36: 345-351. [Crossref]

4. Kei T, Toshiaki O, Shiro N, Megumi W, Yuki Y, et al. (2005) Neutrophilic involvement in the damage to coronary arteries in acute stage of Kawasaki disease. Pediatrics International 47: 305-310. [Crossref] 
5. Patel AS, Bruce M, Harrington W, Portman MA (2015) Coronary artery stenosis risk and time course in Kawasaki disease patients: experience at a US tertiary pediatric centre. Open Heart 2: e000206.

6. Kim NY, Choi DY, Jung MJ, Jeon IS (2008) A case of refractory Kawasaki disease complicated by peripheral ischemia. Pediatr Cardiol 29: 1110-1114. [Crossref]

7. Rowley AH, Baker SC, Shulman ST, Rand KH, Tretiakova MS, et al. (2011) Ultrastructural, immunofluorescence, and RNA evidence support the hypothesis of a "new" virus associated with Kawasaki disease. J Infect Dis 203: 1021-1030. [Crossref]

8. Portman MA, Wiener HW, Silva M, Shendre A, Shrestha S, et al. (2013) DC-SIGN gene promoter variants and IVIG treatment response in Kawasaki disease. Pediatr Rheumatol J 11: 32. [Crossref]

9. Shrestha S, Wiener HW, Aissani B, Shendre A, Tang J, et al. (2015) Imputation of class I and II HLA loci using high-density SNPs from ImmunoChip and their associations with Kawasaki disease in family-based study. Int J Immunogenet 42: 140-146. [Crossref]

Copyright: $\odot 2016$ Hsu B. This is an open-access article distributed under the terms of the Creative Commons Attribution License, which permits unrestricted use, distribution, and reproduction in any medium, provided the original author and source are credited. 